\title{
Rolle und Entwicklung politischer Beratungs- und Begleitgremien nach dem Konzept des Science-Policy Interfaces
}

\author{
Verstärkte Tendenzen zur Erzeugung sozial robusten Wissens \\ in der bundesdeutschen Entsorgung hochradioaktiver Abfälle?
}

Ana María Isidoro Losada, Dörte Themann, Maria Rosaria Di Nucci

\section{Zusammenfassung}

In diesem Beitrag wird eine vergleichende Analyse der Arbeit ausgewählter Kommissionen und Beratungsgremien in der Bundesrepublik Deutschland vorgenommen. Betrachtet werden Kommissionen und Beratungsgremien, die im Verlauf der letzten 40 Jahre den Bundestag und die involvierten Ministerien im Themenfeld des Umgangs mit hochradioaktiven Abfällen beraten haben. Im Mittelpunkt der Science-Policy-Interface-Analyse steht dabei die Rolle der Wissenschaft in Gesellschaft und Politik. Im Fokus des Interesses stehen neben einer genaueren Untersuchung der Zusammensetzung ausgewählter Kommissionen und Beratungsgremien Kriterien wie "Distanz, Pluralität und Transparenz der jeweiligen Instanzen sowie die Empfehlungen für Entsorgungskonzepte und-strategien. FünfGremien werden exemplarisch betrachtet: die Enquete-Kommission "Zukünftige Kernenergiepolitik«, der Arbeitskreis Endlagerung (AkEnd), die Entsorgungskommission (ESK), die Endlager-Kommission (EndKo) und das Nationale Begleitgremium (NBG). Durch die Untersuchung der Arbeit dieser Kommissionen sondieren wir die Rolle wissenschaftlicher Berater*innen und Beratungsgremien in politischen Entscheidungsprozessen. Schlussendlich diskutieren wir die Entwicklung dieser fünf Kommissionen im Zeitverlauf. ${ }^{1}$ wie folgt erschienen ist: Isidoro Losada, Ana M.; Themann, Dörte; Di Nucci, M. Rosaria (2019): Experts and Politics in the German Nuclear Waste Governance. Advisory Bodies between Ambition and Reality. In: Brunnengräber, Achim; Di Nucci, M. Rosaria (2019): Conflicts, Participation and Acceptability in Nuclear Waste Governance. Springer VS, 231- 261. 


\section{Einleitung}

Es existieren gute Gründe für Regierungen, Beratungskommissionen und -gremien für spezifische politische Themen und Herausforderungen einzusetzen. Bei einer mit Unsicherheiten behafteten Entscheidung ist oftmals das Hauptanliegen, das spezifische Fachwissen von ausgewählten Expert*innenkreisen in Anspruch zu nehmen, um fundiertere Entscheidungen treffen zu können. Im Allgemeinen umfassen Beratungsgremien eine Gruppe von Personen, denen eine »epistemische Autorität« (Kruglanski 1980) $)^{2}$ beigemessen wird und die durch das Parlament oder andere Institutionen berufen werden, um eine bestimmte Funktion zu erfüllen. Im Falle parlamentarischer Gremien oder von Untersuchungsausschüssen sind diese Gruppen beauftragt, spezifische Sachverhalte zu untersuchen und ihre Ergebnisse und Empfehlungen dem Gesetzgeber zu übermitteln (Deutschen Bundestag 2019: a, b). Im Unterschied zu solchen parlamentarischen Gremien werden sogenannte Expertenkommissionen oder -gremien ins Leben gerufen, um Wissen und spezifische Expertise zu bündeln, die in dieser Form nicht ohne Weiteres verfügbar wäre. Expertenkommissionen werden idealerweise aus unabhängigen Mitgliedern zusammengesetzt und von einem eigens dafür eingesetzten Sekretariat unterstützt. Im Falle soziotechnischer Belange wird von Kommissionen erwartet, technische und/oder anderweitige Empfehlungen bereitzustellen und ein tieferes Verständnis für bspw. soziotechnische Herausforderungen zu gewinnen. Idealerweise sollte eine pluralistisch zusammengesetzte Expertengruppe angestrebt werden, die ein breites Spektrum an Wissen abdeckt und verschiedene Stakeholdergruppen repräsentiert, um so dem Parlament, den Ministerien bzw. Institutionen eine umfassende Beratung bereitzustellen.

In diesem Beitrag wird eine vergleichende Analyse der Zusammensetzung, Arbeit und Empfehlungen zu Entsorgungskonzepten ausgewählter Kommissionen, die den Deutschen Bundestag hinsichtlich der Endlagerung hochradioaktiver Abfälle beraten haben oder dies weiterhintun, vorgenommen. Die fünf untersuchten Beratungs- und Begleitgremien sind: die Enquete-Kommission Zukünftige Kernenergiepolitik (197983), der Arbeitskreis Endlagerung (AkEnd) (1999-2002), die Entsorgungskommission (ESK) (2008-heute), die Endlager-Kommission (EndKo) (2014-2016) und das Nationale Begleitgremium (NBG) (2016-heute). Auftrag von zwei dieser Gremien (AkEnd und EndKo) war es, hinsichtlich der Auswahl der Entsorgungsoption und der Ausgestaltung des Endlagersuchprozesses beratend tätig zu sein. Das NBG wurde eingerichtet, um die Arbeit der Aufsichtsbehörde sowie der Vorhabenträgerin hinsichtlich des Standortauswahlprozesses kritisch zu begleiten. Im Gegensatz dazu handelt es sich bei der Enquete-Kommission Zukünftige Kernenergiepolitik (nachfolgend: EnqueteKommission), ebenso wie bei der ESK, um Beratungskommissionen, die vom Deutschen Bundestag oder dem Bundesumweltministerium mit dem Ziel eingesetzt wur-

Zürn (2012: 51-52) differenziert zwischen zwei Typen: eine »rein epistemische Autorität«, die auf Fachwissen und moralischer Integrität fußt und eine »politisch zugewiesene epistemische Autorität«, bei der die Zuweisung bzw. Ernennung durch politische Institutionen erfolgt. Zum ersten Typ epistemischer Autorität können beispielswiese NCOs gezählt werden, zum zweiten Typ einberufene wissenschaftliche Beratungsgremien wie (Ethik-) Kommissionen und Sachverständigenräte. 
den, aktuelle technisch-wissenschaftliche Erkenntnisse zum Umgang mit hochradioaktivem Abfall, der Zwischenlagerung und Endlagerung vorzulegen und im Falle der Enquete-Kommission auch zu bewerten.

In unserer Analyse betrachten wir sowohl die kontextuellen und politischen Aspekte ihrer Einberufung als auch verschiedene zeitliche Phasen in einer fast 40 Jahre andauernden Debatte zur Entsorgung hochradioaktiver Abfälle. Einleitend führen wir in die wesentlichen Aspekte des Science-Policy-Interface-Konzeptes (SPI) ein und wenden dieses auf den Kontext der Endlagerungspolitik an. Im nächsten Schritt untersuchen wir anhand von vorab definierten Kriterien die Zusammensetzung und Arbeit der ausgewählten Kommissionen, um im Schlussteil die Entwicklungen beratender Gremien und ihre Bedeutung für politische Entscheidungsfindung herauszustellen.

\section{Wissenschaft - Politik - Gesellschaft: Die Rolle wissenschaftlicher Beratung im politischen Setting}

Im Folgenden werden die Rollen wissenschaftlicher Berater*innen und Beratungsgremien in der Politikgestaltung näher beleuchtet. Unsere Ausgangsthese geht von einem demokratischen Dilemma aus: Entscheidungsträger*innen benötigen oftmals spezifisches wissenschaftliches Wissen sowie kondensierte relevante Informationen und Daten, um fundierte Entscheidungen treffen zu können. Dabei besteht jedoch die Gefahr, dass den Expertenempfehlungen bei der Entscheidungsfindung ein so hohes Maß an Bedeutung beigemessen wird, dass Laienwissen sowie Bürger*innenbeteiligung und -einfluss auf Debatten und Entscheidungen ohne Relevanz bleiben.

Als analytische Grundlage stellen wir verschiedene theoretische Zugänge vor, mit denen die Bedeutung von wissenschaftlicher Beratung, Expert"innenwissen und anderen Wissensformen (Laienwissen, lokales Wissen) im Rahmen von politischen Entscheidungsprozessen umrissen werden kann. Ziel ist es, die Wechselbeziehungen zwischen diesen Aspekten herauszuarbeiten und offenzulegen, inwiefern sie Einfluss auf die Robustheit und Legitimität von Politikgestaltung nehmen. Ferner wird ihre jeweilige Bedeutung in den verschiedenen Phasen politischer Entscheidungsprozesse betrachtet. Schließlich werden diese theoretischen Überlegungen auf den Kontext der Entsorgung hochradioaktiver Abfälle übertragen.

\section{Science Policy Interfaces: theoretische Herausforderungen an den Schnittstellen zwischen Wissenschaft und Politik}

Die gegenwärtigen Ausführungen um Science Policy Interfaces (SPI) rücken Fragen hinsichtlich der Rolle von Wissenschaft in Gesellschaft und Politik in den Fokus. Vertiefende Studien zu den SPI wurden insbesondere von den Politischen Wissenschaften (Boswell 2012, Douglas 2009, Maasen und Weingart 2009) sowie den Science and Technology Studies (STS) vorgelegt (Jasanoff 2004, 2011). Dabei haben Forschungszugänge der STS, bspw. Interessentheorie und Sozialkonstruktivismus, einen genaueren Einblick in die Interdependenzen zwischen Wissenschaftler*innen, wissenschaftlichen Berater*innen 
und anderen politischen Akteuren ermöglicht. »Interest theory traces how the concerns of various actors are embodied in knowledge (...), and social constructionism demonstrates how actors attribute objectivity or fact status to the resulting knowledge through social processes (...).«(Cozzens und Woodhouse 1995: 535f.)

Überlegungen zum SPI kreisen oftmals um die Annahme, dass sich in einer modernen Governancestruktur das Verhältnis von Wissenschaft und Politik in einem Prozess des wechselseitigen aufeinander Einwirkens entwickelt. Wissenschaft per se stellt bereits einen sozialen Prozess dar, der in einen spezifischen sozialen Kontext eingebettet ist und bestimmte Akteure und Institutionen involviert. Entsprechend ist wissenschaftliches Wissen durch soziale Praktiken, Identitäten, Normen, Diskurse und Narrative geformt (Jasanoff 2004: 3). Darüber hinaus kann das SPI nicht nur als Interaktion zwischen Wissenschaft und Politik in Politikprozessen verstanden werden. Es umfasst auch die Interaktion mit weiteren Akteuren etwa aus der Wirtschaft oder der Gesellschaft. In diesem Kontext ermöglichen ko-evolutionäre bzw. gemeinsame Konstruktionen von Wissen, dass Entscheidungsprozesse und Entscheidungen auf Basis einer breiteren Wissen- und Informationsgrundlagegestaltet und getroffen werden (cf. also van den Hove 2007; Jasanoff 2004).

Die Erzeugung von Wissen ist in Praktiken staatlichen Handelns integriert und umgekehrt: Die Art und Weise, wie politische Entscheidungsprozesse gestaltet sind, beeinflusst auch die Wissensproduktion und ihre Nutzung. In der Konsequenz bedeutet das: Wissen im Dienst der Politik ist niemals apolitisch (Jasanoff 2004: 3; 2011: 21). Wissen wird häufig abgerufen, um Orientierung und ein tieferes Verständnis von Zusammenhängen zu gewinnen. Folgt man jedoch den Ausführungen von Karl Popper, so sollte man wissenschaftlich produziertes »objektives« Wissen nicht mit ultimativer Wahrheit verwechseln. Und in der Praxis ist das angeblich objektive Wissen auch immer verschränkt mit subjektivem Wissen und somit sozial und politisch geprägt (vgl. Popper 1963).

»In short, government-funded science is »political«not only in Easton's (1958) sense of authoritatively allocating public resources but also in the sense of exerting symbolic authority by directly or indirectly shaping the ways people think about who they are and what is the real and important..." (Cozzens und Woodhouse 1995: 540).

Cozzens und Woodhouse sehen auch eine Verflechtung zwischen Wissenschaft und Politik hinsichtlich der Herausbildung von Ressourcenkoalitionen (resource coalition) zwischen Wissenschaftler*innen und Regierungsinstitutionen sowie politischen Akteuren (1995: 536). Der Umstand, dass Wissenschaft auf besondere Weise auf staatlich-finanzierte Forschungsförderung und Drittmittelprojekte angewiesen ist, macht sie auch empfänglich für politische Einflussnahme (ausführlicher siehe Guston 2000). Durch diese Abhängigkeit besteht nicht nur die Gefahr, dass die Forschungsagenda in eine bestimmte Richtung gelenkt, sondern auch die Unabhängigkeit wissenschaftlicher Prozesse durch öffentlich finanzierte Forschungsprojekte untergraben werden kann.

Forderungen aus der Gesellschaft nach einer neutralen oder "sachlich angemessenen« politischen Entscheidungsfindung machen eine adäquate Unterstützung von politischen Entscheidungsprozessen durch wissenschaftliche evidenzbasierte Ergebnisse notwendig (Jasanoff 1990: 241). Dennoch kann evidenzbasierte Politik manchmal Kon- 
flikte hervorrufen, denn das Maß an Evidenz und was eigentlich eine ausreichende Evidenz bedeutet, sind umstritten, genauso wie die Frage, ob verschiedene politische Schritte im Entscheidungsprozess eine unterschiedliche Evidenzbasis benötigen (Pedersen 2014: 548).

\section{Rolle von Beratungsgremien in politischen Entscheidungsprozessen}

Neben wissenschaftlichen Ansprüchen wie Evidenz und Validität müssen Beratungsgremien zusätzlich Prozesse der Deliberation befolgen und demokratische Politikgestaltung respektieren (Pedersen 2014: 548). Die Einsetzung eines Beratungsgremiums ist vor allem wichtig für solche politischen Entscheidungsprozesse, in denen Wissen als unsicher und mehrdeutig gilt. Abb.1veranschaulicht vier verschiedene Gründe, weswegen eine Expertenkommission einberufen werden kann. Neben dem Ziel der Bereitstellung von Wissen und Klärung von Fakten, kann die Einberufung von beratenden Gremien auch durchaus aus prozessbezogenen und handlungspolitischen Gründen erwogen werden.

Abbildung 1: Motive zur Einberufung einer

Expertenkommission

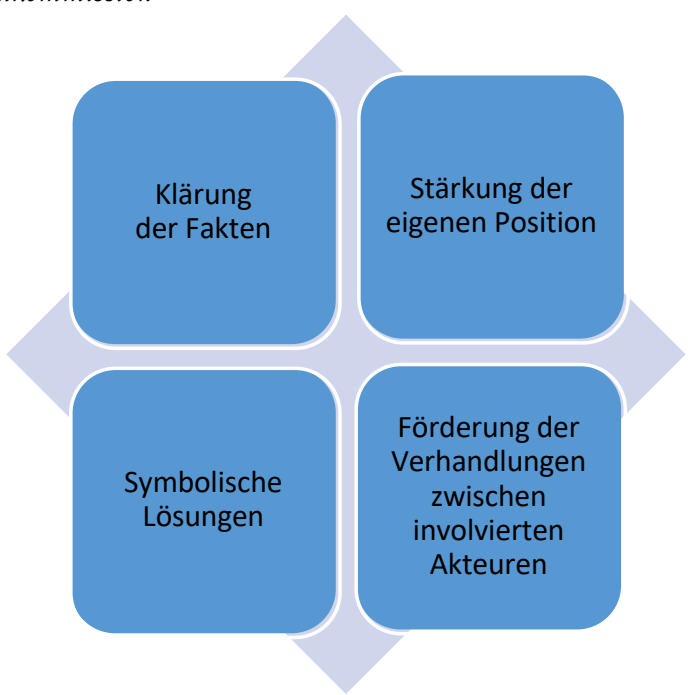

Quelle: Eigene Darstellung basierend

auf Siefken 2007: 78.

Die Berücksichtigung und der Einbezug von gesellschaftlichen Akteuren sowie von lokalem Wissen ist ein wichtiger Schritt, um sozial robustes Wissen im Sinne einer »kontext-sensiblen « Wissenschaft (Gibbons 2000) ${ }^{3}$ zu erzeugen. Eine grundlegende Er- 
kenntnis des sogenannten participatory turn (Bergmans et al. 2015) im Kontext des Managements hochradioaktiver Abfälle verdeutlicht, dass Anti-Atomkraft-Bewegungen, Umweltorganisationen sowie die durch eine politische Entscheidung betroffene Bevölkerung als eine Art »countervailing force to the knowledge/power alliance« (Cozzens und Woodhouse 1995: 535) agieren können.

Akteure aus der Wissenschaft sollten offen für inhaltliche Auseinandersetzung sein und die jeweiligen Gruppen einbeziehen, die durch eine wissenschaftsbasierte politische Entscheidung betroffen sind oder sein könnten (Pedersen 2014: 548). Eine solche Handlungsweise würde das Ideal der context-sensitive science widerspiegeln, wie sie Gibbons $(1999,2000)$ auf einer Mikroebene politischen Entscheidens definierte. Auf diese Weise, so Gibbons, würde die Gesellschaft zur Wissenschaft sprechen (society is speaking back to science) (1999: 82). Zu beachten ist hierbei, dass die Interaktion zwischen wissenschaftlichen Expert*innen und Entscheidungsträger*innen als ein institutioneller Mechanismus angesehen werden kann, bei dem zwei verschiedene politische Begründungsformen, epistemische und politische Robustheit, zusammenwirken sollen. Um einen hohen Beratungsstandard zu gewährleisten bzw. Vertrauenswürdigkeit zu generieren, sind beide Dimensionen erforderlich (Lentsch und Weingart 2011: 8).

Beratungsgremien, die mit der Lösung eines schwierigen politischen oder sozialen Problems betraut sind, müssen vor allem epistemische Robustheit erzeugen. Dies stellt aber gewisse Ansprüche an die Qualität des in Gremien erzeugten Wissens. Da das Wissen, das in diesem Kontext erzeugt wird, ein politisches Problem aufgreifen soll, muss die Expertise über das rein wissenschaftlich-disziplinäre Denken hinausgehen und anderen Anforderungen an Exaktheit und Validität entsprechen, als die wissenschaftliche Forschung (ebd.). Wenn Wissen von Unsicherheit oder Mehrdeutigkeit geprägt ist (siehe auch Siefken 2007), dann kann Robustheit erzeugt werden, »if the probabilities of the postulated functional and causal relations are reliable." (Lentsch und Weingart 2011: 8) Für die Entscheidung über ein Thema, das von Unsicherheit und begrenzter wissenschaftlicher Evidenz geprägt ist, bedarf es entsprechend eines legitimen Prozesses, der verschiedene Typen von Wissen einbezieht, um diesen politisch robust zu führen.

Dies knüpft an die genannte politische Robustheit von Wissen an, welche die Akzeptabilität von Wissen und den Einbezug verschiedener Wissenstypen in Beratungsgremien reflektiert. Der Rat durch eine Kommission wird dann robust, wenn die Empfehlungen den Kriterien und Normen von Entscheidungsträger"innen entsprechen und in ihrer Umsetzung durchführbar sind (Lentsch und Weingart 2011: 8). Daneben sind für politische Robustheit aber auch das (lokale) Wissen und Interessen sowie Präferenzen der betroffenen Stakeholder und Bürger*innen einzubeziehen (Bergmans et al. 2015; Lentsch und Weingart 2011; Pedersen 2014).

Die genannten Konzepte und Mechanismen sind wichtig für die kritische Untersuchung von Beratungsgremien. Obwohl Pedersens social contract (2014) zeigt, dass wissenschaftliches Wissen und Fakten die wesentliche Basis für die Legitimität politischer

bust knowledge, that is, knowledge likely to be reliable not only inside but also outside the laboratory.« (Gibbons 2000: 161) Durch ein verstärkt interaktives Verhältnis zwischen Wissenschaft und Gesellschaft wird laut Gibbons (2000) somit ein robusteres Wissen erzeugt, dadurch, dass es stärker gesellschaftlich eingebettet und im Vergleich weniger stark umstritten ist. 
Entscheidungen darstellen, so kann politische Legitimität dennoch nicht allein durch den Einfluss von Wissenschaft auf den Entscheidungsprozess erzeugt werden. Mit Blick auf robuste Entscheidungsfindungen sind verschiedene Faktoren von Bedeutung, wie etwa die Nachvollziehbarkeit von Wissen, die Relevanz von bestimmtem wissenschaftlichen Wissen für politische Entscheidungen und Legitimität (vor allem verstanden als der Einbezug von Werten und Perspektiven derjenigen Gruppen, die durch die Entscheidung betroffen sind) (Lentsch und Weingart 2011: 7). In gewisser Weise wird Legitimität noch durch Transparenz und Vertrauenswürdigkeit bzw. Seriosität der wissenschaftlichen Beratung verstärkt. Darüber hinaus werden Robustheit, Legitimität und Glaubwürdigkeit stark durch historische und nationale Kontexte beeinflusst.

Aus dieser Annahme heraus wird pluralistischen (und konfliktiven) Beratungssystemen eine besondere Rolle zugewiesen. Sie erhalten den Auftrag, eine wissenschaftsorientierte Politikgestaltung zu konzeptualisieren (Cozzens und Woodhouse 1995: 543), welche zugleich die vielfältigen öffentlichen Bedenken ernst nimmt und aufgreift.

Bei der Zusammensetzung und inhaltlichen Fokussierung von wissenschaftlichen Beratungsgremien könnte idealerweise berücksichtigt werden, dass es keine gleichwertige Konkurrenz zwischen verschiedenen gesellschaftlichen Interessen gibt, da bspw. verschiedene Akteursgruppen unterschiedliche Organisationsniveaus, Ressourcen und Kompetenzen besitzen, um ihre Interessen zu artikulieren. Cozzens und Woodhouse (1995) beschreiben es als Aufgabe von Beratungsgremien, diese Ungleichheit in politischen Aushandlungsprozessen ebenso wie die gelegentlich vertretene Ansicht unter Wissenschaftler*innen, »that the public is neither interested nor competent in the governmental matters scientists deal with (Cozzens und Woodhouse 1995: 546), zu überwinden. Es besteht also ein Bedarf an kritischer Betrachtung der Zusammensetzung von Beratungsgremien, die sowohl die Vielfalt an Disziplinen, aber auch den Einbezug verschiedener Stakeholdergruppen und Wissensformen prüft.

\section{Die Rolle von Kommissionen und Beratungsgremien in der bundesdeutschen Endlagerpolitik}

Seit 1969 hat der Deutsche Bundestag die Möglichkeit, Enquete-Kommissionen »zur Vorbereitung von Entscheidungen über umfangreiche und bedeutsame Sachkomplexe« einzuberufen (Deutscher Bundestag 2017, \$56 (1)). In Übereinstimmung mit der Geschäftsordnung und anderen Rechtsvorschriften sind in den vergangenen Jahrzehnten verschiedene Gremien und Kommissionen einberufen worden, um Empfehlung für das deutsche Standortauswahlverfahren zu erarbeiten. In diesem Abschnitt kontextualisieren wir fünf Gremien bzw. Kommissionen hinsichtlich der Anlässe ihrer Einsetzung, der Zugehörigkeit ihrer Mitglieder, der inhaltlichen Schwerpunktsetzung, den formulierten Empfehlungen und dem Austausch mit anderen Akteuren und Institutionen.

\section{Enquete-Kommission "Zukünftige Kernenergiepolitik » (1979-83)}

1979 entschied der Deutsche Bundestag die Einrichtung der Enquete-Kommission Zukünftige Kernenergiepolitik (atommüllreport 2016; Deutscher Bundestag 1980). Die Ar- 
beit und die Agenda dieser Kommission lässt sich in zwei Perioden einteilen, da die vormalige Regierung durch die Bundestagswahlen 1980 abgelöst wurde und die Kommission ein erneutes Votum des Bundestages benötigte, um weiterarbeiten zu können. Diese Unterbrechung sorgte für eine neue thematische Fokussierung. Da die Entsorgung hochradioaktiver Abfälle explizit im Rahmen der ersten Arbeitsphase (1979-80) thematisiert wurde, konzentriert sich unsere Analyse auf die Zusammensetzung und inhaltliche Fokussierung während dieser ersten zwei Jahre sowie auf den ersten Bericht von 1980.

Die Kommission setzte sich aus 15 Mitglieder zusammen, von denen sieben dem Parlament angehörten und acht als externe Experten einbezogen wurden. Das Arbeitsprogramm war in sieben Themenfelder untergliedert. Eines davon sollte die Voraussetzungen für ein optimales Entsorgungskonzept hochradioaktiver Abfälle in der Bundesrepublik spezifizieren. In ihren Schlussfolgerungen resümierte die Enquete-Kommission, dass die Akzeptanz der Atomenergienutzung eng an die Frage nach der Entsorgung abgebrannter Brennelemente genknüpft sei. Neue Kernkraftwerke könnten nur in Betracht gezogen werden, sofern eine sichere Entsorgung garantiert werden könne (Deutscher Bundestag 1980: 163).

Die abschließenden Empfehlungen der Kommission zu diesem Themenkomplex lauteten:

a) Die Realisierung einer Zwischenlagerlösung für die nahe Zukunft - mit Fokus auf die Trockenlagerung - muss vorangetrieben werden. Diese Lagerungsoption kann als rückholbar angelegt werden, z.B. in Untergrundlagern;

b) Testbohrungen und vergleichbare Standards sind notwendig, um die Eignung von Gorleben und anderer optionaler Standorte festzustellen;

c) jede Prüfung muss eine umfängliche Information für Bürger*innen und die Beteiligung von Wissenschaftler*innen garantieren, die die Eignung des betreffenden möglichen Endlagers kritisch überprüfen;

d) die Option der Rückholbarkeit von gelagerten Abfällen sollte bei der Beforschung von Lagerungsoptionen und Wiederaufbereitung berücksichtigt werden.

\section{Arbeitskreis Auswahlverfahren Endlagerstandorte (AkEnd) (1999-2002)}

Im Jahr 1999 setzte das Bundesumweltministerium (BMU) den Arbeitskreis Auswahlverfahren Endlagerstandorte (AkEnd) ein. Das Ziel dieses Gremiums war es, neue wissenschaftsbasierte und gesellschaftliche Standortauswahlverfahren $\mathrm{zu}$ identifizieren, die eine offene und transparente Suche für ein Endlager ermöglichen. Zu diesem Zweck definierte der Arbeitskreis Auswahlkriterien sowie notwendige Verfahrensschritte. Eine große Neuerung war in diesem Zusammenhang, dass der empfohlene Ansatz nicht nur auf technischen Aspekten beruhte, sondern auch sozialwissenschaftliche Kriterien berücksichtigte (AkEnd 2002). Dieser neue Fokus entsprach den spätestens seit Ende der 1990er Jahre in Gang gesetzten Entwicklungen, in denen es zu einer verstärkten Öffnung gegenüber Beteiligungsverfahren kam und die Notwendigkeit der Bürger*innenbeteiligung in verschiedenen endlagerbezogenen Entscheidungsprozessen formuliert wurde (Bergmans et al. 2015). Der AkEnd schlug ein Drei-Phasen-Modell zur Su- 
che eines Endlagers vor. In einer ersten Phase erfolgt die Festlegung von Kriterien für die Standortauswahl und das Suchverfahren. In der zweiten Phase sollten das vorgeschlagene Auswahlverfahren und dessen Kriterien mit verschiedenen Stakeholdern erörtert werden. In der dritten Phase sollte das Verfahren dann umgesetzt werden. Dieses schrittweise Vorgehen wurde auch gewählt, um Transparenz und klare Verantwortlichkeiten im Prozess sicherzustellen (AkEnd 2002: 69). Aufgrund der intensiven gesellschaftlichen Auseinandersetzungen und dem hohen Vertrauensverlust in staatliche Akteure im Rahmen der Entsorgung hochradioaktiver Abfälle empfahl der AkEnd erstmals einen umfassenden gesellschaftlichen Beteiligungsprozess für das Standortsuchverfahren.

\section{Die Entsorgungskommission (ESK) (2008 - bis heute)}

Die Entsorgungskommission (ESK) wurde 2008 durch das BMU einberufen. Sie wird durch eine Geschäftsstelle unterstützt, die beim Bundesamt für die Sicherheit der nuklearen Entsorgung (BASE) angesiedelt, diesem gegenüber jedoch weisungsunabhängig ist. Die ESK berät das BMU bezüglich aller Sachlagen, die den Umgang mit hochradioaktiven Abfällen (Konditionierung, Lagerung und Transport radioaktiver Materialien, die Stilllegung und den Rückbau nuklearer Anlagen sowie Entsorgung in tiefen geologischen Formationen) betreffen. Sie unterbreitet (natur-)wissenschaftliche und technische Empfehlungen oder formuliert Stellungnahmen. Sie gibt allerdings keine politischen oder rechtlichen Einschätzungen ab. Derzeit setzt sich die ESK aus 13 Mitgliedern zusammen, die allesamt einen naturwissenschaftlichen Hintergrund in Geologie, Chemie oder Physik haben oder aber aus dem Bereich Maschinenbau kommen. Das BMU benennt die Mitglieder für die Dauer von drei Jahren, mit Ausnahme des Vorsitzes, den seit der Gründung der ESK 2008 bis Anfang 2020 die gleiche Person innehatte. Die Arbeit der ESK ist nicht öffentlich und neben den Mitgliedern dürfen nur (Verwaltungs-)Angehörige des BMU an den Sitzungen teilnehmen. Vertreter*innen von weiteren Stakeholder-Gruppen wie regionale Behörden, Expert"innen, Betreiber und kritische Zivilgesellschaft können an bestimmten Themensitzungen der ESK teilnehmen. Die ESK organisiert sich in Arbeitsgruppen und Ausschüssen, die sich mit spezifischen Aspekten des Managements hochradioaktiver Abfälle beschäftigen. In diesem Rahmen etablierte die ESK auch den Ausschuss Endlagerung radioaktiver Abfälle (EL). Zwischen 2014 und 2015 evaluierte dieser Ausschuss die Kriterien für den Standortauswahlprozess wie er durch den AkEnd vorgeschlagen wurde. Der EL bekräftigte die meisten der Empfehlungen und Ergebnisse des AkEnd und empfahl zudem die Endlagerung hochradioaktiver Abfälle in tiefen geologischen Formationen (ESK 2015).

\section{Kommission "Lagerung hoch radioaktiver Abfallstoffe» (EndKo) (2014-2016)}

Die Kommission Lagerung hoch radioaktiver Abfallstoffe (EndKo) wurde 2014 durch den Bundesrat und den Bundestag eingesetzt (EndKo 2016). Ziele und Aufgaben der Kommission wurden durch das StandAG von 2013 definiert. Hauptaufgabe war die Ausarbeitung wesentlicher Prinzipien der Entscheidungsfindung mit Blick auf den Auswahl- 
prozess, die Entwicklung von Kriterien, die bei der Auswahl angewandt werden sollten, sowie die Ausgestaltung des Prozesses hinsichtlich der Bürger*innenbeteiligung.

Die EndKo setzte sich aus 32 Mitglieder sowie zwei Vorsitzenden zusammen. Die Mitglieder wurden aus verschiedenen Stakeholder-Gruppen ausgewählt, zu denen die Wissenschaft, Zivilgesellschaft (hier Gewerkschaften, Kirchen, Umwelt-NGO und Industrie) sowie Mitglieder des Bundesrates und des Bundestages zählten. Wobei die Vertreter*innen der beiden letzteren Gruppen keine Stimmrechte in der Kommission hatten und somit nur ihre Positionen in die Debatten einbringen konnten.

Mit Blick auf die entwickelten Inhalte und Empfehlungen weisen die Berichte der EndKo und des AkEnd substanzielle Überschneidungen auf. Dies wird vor allem bei dem Vergleich der empfohlenen Auswahlkriterien der beiden Kommissionen deutlich, die im Wesentlichen deckungsgleich sind (für weitere Details siehe: AkEnd 2002: 205 und EndKo 2016: 48f.). Für beide Kommissionen stellten Sicherheit und Risiko zentrale Aspekte der Endlagerstandortsuche dar (EndKo 2016: 26ff.). Folglich spielten naturwissenschaftliche und technisch-geologische Kriterien eine vorrangige Rolle bei der Identifizierung potenzieller Endlagerstandorte. Analog zum AkEnd schlug die EndKo ein Auswahlverfahren vor, in dem eine umfassende Öffentlichkeitsbeteiligung vorgesehen sein sollte und die über die gesetzlich vorgeschriebenen Mindestanforderungen in diesem Bereich hinausgehen. In diesem Sinne hat die EndKo ein Konzept für Bürger*innenbeteiligung in der Standortauswahl entwickelt, welches im Detail die wesentlichen Beteiligungsaspekte definiert.

Darüber hinaus empfahl die EndKo die Endlagerung im tiefengeologischen Untergrund als die präferierte Entsorgungsoption. Im Unterschied zum AkEnd sprach sich die EndKo jedoch für die explizite Möglichkeit der Rückholbarkeit der Abfälle aus. Sie empfahl die Option der Bergbarkeit für die ersten 500 Jahre nach der Einlagerung, sowie dass der gesamte Endlagerungsprozess als ein (selbst-)lernender Prozess konzipiert werden sollte, der auf einer neuen Fehlerkultur basiert. Diese sollte durch die Möglichkeit zu Rücksprüngen im Prozess und die Reversibilität von Entscheidungen innerhalb des Prozesses geprägt sein (ebd.: 31ff.).

\section{Das Nationale Begleitgremium (NBG) (2016 bis heute)}

Das Nationale Begleitgremium (NBG) wurde 2016 in Anlehnung an die Empfehlungen der EndKo durch das BMU gegründet. Das NBG ist an das Umweltbundesamt (UBA) und somit auch an dessen Verwaltungsstrukturen angegliedert. Die Aufgaben, Rechte und Pflichten des NBG werden in $\$ 8$ des StandAG in der überarbeiteten Fassung von 2017 festgelegt. Die Gründung eines solchen beaufsichtigenden Gremiums war bereits Teil des Empfehlungskataloges des AkEnd (AkEnd 2002: 2 und 207). Das NBG basiert auf einem neuen Beteiligungsformat, das verbunden ist mit dem empfohlenen Beteiligungskonzept der EndKo (EndKo 2016: 383ff.). Die EndKo betrachtete hierbei das NBG als integralen Teil eines erweiterten und lernfähigen Beteiligungssystems (ebd.: 40).

Zentrale Aufgabe des NBG ist die kritische Begleitung der Arbeit der Aufsichtsbehörde BASE, sowie des Betreibers der Bundesgesellschaft für Endlagerung (BGE), hinsichtlich des Standortauswahlprozesses. Dies beinhaltet vor allem, im Prozess zu vermitteln und den öffentlichen Beteiligungsprozess zu begleiten. In diesem Sinne hat 
das Gremium eine Vermittlungs- und Beobachtungsaufgabe bezüglich der Beteiligung der Öffentlichkeit am Standortauswahlprozess (NBG 2016).

Das NBG ist in seiner Zusammensetzung pluralistisch. Es soll 18 Mitglieder umfassen, davon sechs Bürgervertreter"innen und zwölfrespektierte Personen des öffentlichen Lebens. Die vollständige Mitgliederzahl erreichte das Gremium schließlich im März 2020. Davor war das Gremium mit zwölf Mitgliedern kontinuierlich unterbesetzt. Die Mitglieder dürfen »keiner gesetzgebenden Körperschaft oder Regierung des Bundes oder eines Bundeslandes angehören. Zudem dürfen sie keinerlei wirtschaftliche Interessen in Bezug auf Standortauswahl oder die Endlagerung haben « NBG 2016, NBG 2020). Das NBG kommt monatlich in meist öffentlichen Sitzungen zusammen. An diesen Sitzungen nehmen Vertreter*innen von BGE und BASE oder anderen relevanten Institutionen regelmäßig teil, um über aktuelle Sachstände und Ergebnisse $\mathrm{zu}$ berichten. Die Aufgaben und das Vorgehen des NBG orientiert sich auf einer neu $\mathrm{zu}$ kultivierenden Fehlerkultur, wie sie zuerst durch den AkEnd vorgeschlagen wurde. Diese soll dazu führen, das Verfahren insgesamt sowie das Vorgehen von BASE und BGE stetig zu hinterfragen und kritisch zu reflektieren.

\section{Analyse der Beratungsgremien unter Berücksichtigung der Aspekte Distanz, Pluralität und Transparenz}

Um die Reichweite und politische Resonanz der Empfehlungen beurteilen und die Arbeit sowie das Design der ausgewählten Gremien und Kommissionen evaluieren zu können, nutzen wir die vier Prinzipien guter wissenschaftlicher Politikberatung, die laut Lentsch und Weingart (2011: 15f.) zur Erreichung epistemischer wie auch politischer Robustheit notwendig einzuhalten sind (siehe Abb. 2).

Zunächst betrachten wir die Gremien unter dem Aspekt des Pluralismus der Mitgliederzusammensetzung. Um diesbezügliche Aussagen treffen zu können, werden verschiedene Kriterien betrachtet: die Vielfalt an Disziplinen, Verhältnis der einbezogenen Disziplinen (z.B. Natur- oder Sozialwissenschaften); Vertretung weiterer Stakeholdergruppen wie Kirchen, Gewerkschaften, NGOs etc.; Einbezug von lokalem und/oder Laienwissen sowie das Verhältnis von männlichen und weiblichen Mitgliedern. All diese Kriterien sind eng verknüpft mit der Eingangsfrage, ob die Gremien/Kommissionen ein breites Wissens- und Interessenspektrum abbilden. Durch die Analyse dieser Kriterien können wir Aussagen darüber ableiten, inwiefern der Entscheidungsprozess die Voraussetzung dafür erfüllt, was wir in Abschnitt 2 als politisch robuste Entscheidungen beschrieben haben.

Die Prinzipien »Transparenz« und »Zugänglichkeit zu Informationen« behandeln wir in einem separaten Abschnitt, in dem wir darauf eingehen, inwiefern die Gremien in der Lage waren, Vertrauen zu erzeugen. Daran anschließend analysieren wir den Einfluss ihrer Empfehlungen auf Entscheidungsprozesse.

Zum Schluss betrachten wir das Prinzip der »Distanz« bzw. »Unabhängigkeit« der Expert*innenberatung. Dieses Prinzip steht in Verbindung mit der Frage, ob das Beratungsgremium spezifische Stakeholder oder bestimmte politische Prioritäten vertritt. 
Abbildung 2: Prinzipien guter wissenschaftlicher Politikberatung

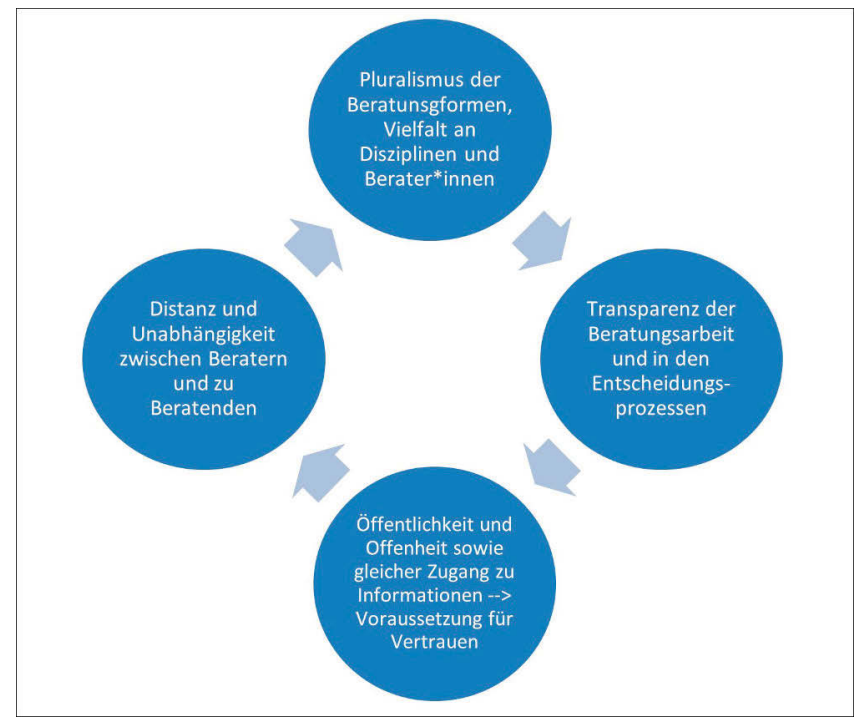

Quelle: Eigene Darstellung basierend auf Lentsch und Weingart 2011

\section{Pluralismus der Beratungsformen und Bandbreite verschiedener Disziplinen und Berater*innen}

Bei der Analyse der disziplinären Zusammensetzung der Beratungsgremien identifizieren wir (außer beim NBG) eine stark naturwissenschaftliche Ausrichtung. Im Falle der Enquete-Kommission sind insbesondere Physiker zahlreich vertreten (was u.a. damit zusammenhängen könnte, dass zum damaligen Zeitpunkt die Wiederaufarbeitung von Atommüll noch als Option zur Diskussion stand). Unter den vier Mitgliedern in der Arbeitsgruppe der Kommission zu Fragen der Entsorgung hochradioaktiver Abfälle waren, mit der Ausnahme von einem Gewerkschafter, ausschließlich Naturwissenschaftler vertreten.

Im AkEnd konnten wir, anders als bei der Enquete-Kommission, eine starke Einbindung von Geologen und Geophysikern beobachten, während andere naturwissenschaftliche Disziplinen wie etwa Physik, Chemie, Bauingenieurwesen oder Mathematik verhältnismäßig geringer vertreten waren. Auch wenn im AkEnd eine deutlich größere disziplinäre Bandbreite vertreten war, zählte nur ein Sozialwissenschaftler (Fachbereich Stadt- und Landschaftsplanung) zu den 14 Mitgliedern.

Die so genannten Ausschluss- und Abwägungskriterien sowie die Mindestanforderungen sind ausschließlich technischer und naturwissenschaftlicher Natur. Auch wenn natur- und sozialwissenschaftliche Kriterien rein formell gleich gewertet werden, sind soziale Kriterien weder Teil der Ausschlusskriterien noch der Mindestanforderungen. Da die regionale Implementierung erst nach der Standortauswahl beginnt, stellen so- 
zialwissenschaftliche Kriterien nur einen sekundären Maßstab zur Bewertung der Teilgebiete dar (AkEnd 2002: 189ff.).

Die ESK setzt sich ausschließlich aus Naturwissenschaftler"innen zusammen. Sie ist zu gleichen Anteilen mit Geolog*innen, Chemiker*innen, Physiker*innen und Maschinenbauer*innen und mit einem/r Mathematiker*in besetzt. Im Hinblick auf den Anspruch dieser Kommission ${ }^{4}$, alle nötigen Disziplinen und das gesamte Spektrum der aktuellen Forschung widerzuspiegeln, um das Umweltministerium bezüglich seines Umgangs mit und der Entsorgung von hochradioaktiven Abfällen beraten zu können, kann festgestellt werden, dass weder Raum- und Regionalplaner*innen noch Sozialwissenschaftler*innen, etwa in Form von Risikoforschung, in der Expert*innengruppe vertreten sind. Außerdem ist es wichtig zu erwähnen, dass dieses Gremium auch die EndKo beraten hat. Des Weiteren war der ehemalige Vorsitzende der ESK bereits Mitglied des AkEnd und gehörte der Gruppe der Wissenschaft in der EndKo an. Jasanoff weist darauf hin, dass Wissenschaft im Allgemeinen meist keine direkten Verbindungen zu Politik und Entscheidungsträger*innen aufweist. Stattdessen bestehen Verbindungen zwischen einzelnen »Expert*innen « oder Berater*innen, die als »a cadre of knowledgable professionals with their own social relations, ethical commitments, and connections to power « (2011: 21) zu verstehen sind.

Mit der Berufung der EndKo ging ein erkennbarer Bruch mit dieser Konzentration auf naturwissenschaftliche Expertise einher. Zusätzlich zu den acht Wissenschaftler*innen wurden acht Vertreter*innen gesellschaftlicher Gruppen wie Gewerkschaften, Kirchen, Umweltverbände, aber auch Energieversorgungsunternehmen einbezogen. Dies wirkt zunächst wie eine positive, den Beratungsprozess öffnende Entwicklung, die Akteure außerhalb üblicher wissenschaftlicher oder die Entsorgung hochradioaktiver Abfälle organisierender Netzwerke (z.B. KIT, GNS, TÜV NORD/SÜD, BGR etc.) eine Stimme gab. Dennoch wurde die zahlenmäßige Überlegenheit der Naturwissenschaftler*innen innerhalb der wissenschaftlichen Gruppe nicht in Frage gestellt. Hinzu kommt, dass drei der acht Wissenschaftler*innen bereits im AkEnd mitgearbeitet hatten. Die Entscheidung, diese drei ehemaligen AkEnd-Mitglieder mit einzubeziehen, kann sowohl positiv im Sinne einer Kontinuität - Übertragung und Fortentwicklung von Wissen und Erfahrungen - oder aber auch negativ im Sinne des Fortschreibens von Pfadabhängigkeiten der AkEnd-Empfehlungen interpretiert werden. ${ }^{5}$ Daneben ist die disziplinäre Zusammensetzung mit Blick auf die, durch das StandAG

»In der ESK sollen die Fachgebiete vertreten sein, die für die sachverständige Beratung des BMU erforderlich sind. Die Mitglieder müssen die Gewähr für eine sachverständige und objektive Beratung bieten. Um eine ausgewogene Beratung sicherzustellen, soll die ESK so besetzt sein, dass die gesamte Bandbreite der nach dem Stand von Wissenschaft und Technik vertretbaren Anschauungen repräsentiert ist. «Siehe: www.entsorgungskommission.de/de/zusammensetzung-esk, zuletzt aufgerufen am 03. August 2020.

5 Die Möglichkeit von Pfadabhängigkeiten wird deutlich bei der Betrachtung von Wortprotokollen des kick-off meetings der EndKo. Dort plädierte ein ehemaliges Mitglied des BfS (Bundesamt für Strahlenschutz und Vorläufer der aktuellen Regulierungsbehörde BASE) für die Beibehaltung von AkEnd-Empfehlungen. (Wortprotokoll: https://www.ausgestrahlt.de/informieren/atommuell/stan dortauswahlverfahren/wortprotokolle/, zuletzt aufgerufen am 28. März 2019). 
definierte, erste Aufgabe der EndKo - die Diskussion und Evaluierung alternativer Optionen im Umgang mit nuklearen Abfällen (StandAG 2013: \$4 Abs. 2.1) - kritisch zu betrachten. Das für eine solche Bewertung notwendige multidisziplinäre Wissen wurde durch die Zusammensetzung der Kommission nicht im erforderlichen Maße gewährleistet. So besaßen die Vertreter*innen der Wissenschaft eindeutige Expertise im Bereich der Geologie, aber keine spezifische Expertise bezüglich alternativer Optionen im Umgang mit hochradioaktiven Abfällen. Diese Beobachtung offenbart einige problematische Aspekte: Ein der Kommissionszusammensetzung geschuldeter Bias zugunsten geologischer Expertise könnte mit einer stärkeren Betrachtung und Befürwortung tiefengeologischer Endlagerungsoptionen einhergehen. Um stattdessen auch alternative Entsorgungsoptionen wie etwa langzeitliche oberflächennahe oder überirdische Lagerung von abgebrannten Brennelementen, tiefe Bohrlöcher oder andere Optionen evaluieren zu können, wäre die Expertise von Bauingenieur"innen, Materialforscher*innen oder etwa Physiker*innen hilfreich gewesen. Verstärkt werden könnte dieser Effekt durch sogenannte resource coalitions. Wissenschaftler*innen mit großer relevanter Expertise in einem bestimmten Umgang mit hochradioaktiven Abfällen könnten ein Interesse haben, zugunsten dieser Option zu beraten, ohne dabei Alternativen zu berücksichtigen, da eine solche, sie begünstigende Ausrichtung der Politik unter anderem auch entsprechende Forschungsgelder freisetzen würde.

Jenseits eines Überhangs an naturwissenschaftlicher Expertise fallen auch die unausgewogenen Geschlechterverhältnisse ins Auge. Weder in der Enquete-Kommission noch im AkEnd waren weibliche Mitglieder vertreten. Die ESK verzeichnete bis Ende 2019 vier weibliche Mitglieder von insgesamt zwölf. Im Januar 2020 wurde eine Frau zur Vorsitzenden gewählt und aktuell weist die Zusammensetzung der ESK ein Verhältnis von fünf weiblichen gegenüber acht männlichen Mitgliedern auf. Auch die Zusammensetzung der EndKo wies kein auch nur annähernd ausgeglichenes Geschlechterverhältnis auf. Unter den wissenschaftlichen Mitgliedern war keine einzige Frau vertreten und aus dem Kreis der Zivilgesellschaft beteiligte sich lediglich eine Gewerkschaftsvertreterin.

Das NBG ist in vielerlei Hinsicht ein Novum. Dies gilt insbesondere für sein Design und seine Rolle in der gesamten Institutionenarchitektur des bundesdeutschen Standortauswahlprozesses. Das NBG hat ein ausgewogenes Geschlechterverhältnis. Darüber hinaus zeigt es sich offen für gesellschaftliche Fragen und Diskurse. Erstmals werden zufällig ausgewählte Bürger*innen (-vertreter*innen) als gleichberechtigte Mitglieder einbezogen. Die Schaffung des NBG spiegelt ebenso eine Entwicklung hin zu einer stärkeren Berücksichtigung anderer Wissensformen wie etwa lokales Wissen, Laienwissen oder der »reinen epistemischen Autorität« (Zürn 2012) durch NGO wider. Dies sind wichtige Schritte hin zu einer Demokratisierung solch risikobehafteter Großinfrastrukturprojekte.

Das NBG fördert den Austausch zwischen den verschiedenen, sich mit hochradioaktiven Abfällen befassenden Institutionen, Behörden, Organisationen und der Zivilgesellschaft. Dies äußert sich bspw. in der Berufung des Partizipationsbeauftragten, der mögliche Spannungen zwischen Stakeholdern im Standortauswahlverfahren ausmachen und unterstützend bei der Bewältigung von Konflikten wirken soll. Daneben lud das NBG im Kontext der Novellierung des StandAG Bürger*innen zur gemeinsa- 
men Erarbeitung von Änderungsvorschlägen ein. Dennoch blieben die Vorschläge des NBG sowie anderer zivilgesellschaftlicher Akteure bei der Novelle des StandAG weitestgehend unberücksichtigt (atommüllreport 2020), was zu Kritik der zivilgesellschaftlichen Akteure führte (Becker 2017; Stay 2019). Insgesamt ist eine Entwicklung zu mehr politischer Robustheit festzustellen, in dem Sinne, dass auch Wissen und Präferenzen der betroffenen Bevölkerung im Laufe der Zeit verstärkt einbezogen werden, jedoch mit verbleibenden Defiziten im Bereich der Rechenschaftslegung der Entscheidungsträger*innen oder im Bereich der Entwicklung hin zu einer kontext-sensiblen Wissenschaft.

Sachverständige, wie die wissenschaftlichen Mitglieder des AkEnd oder der EndKo, stellen keineswegs eine homogene Gruppe dar, allerdings gehören sie größtenteils einer (wissenschaftlichen) Community an. Eine solche Community zeichnet sich dadurch aus, dass seine Mitglieder eine sehr spezifische Ausbildung genossen haben, die sie da$\mathrm{zu}$ prädestiniert, mit ähnlichen Methoden und Zielen zu arbeiten und gleichzeitig eine gemeinsame Wahrnehmung und Sicht auf die Welt zu teilen. »It is chiefly this shared perception that unites the members of the community and helps to define their paradigm.« (Di Nucci und Pearce 1989: 417) Je mehr Kommissionen offen sind für Wissen und Expertise jenseits akademischer Wissenschaft und je mehr sie Praktiker*innen, Vertreter*innen der Industrie und der Gesellschaft mit einbeziehen, desto mehr werden ihre Wahrnehmungen variieren und teils in Konflikt geraten, aber auch eine höhere Kontext-Sensibilität im Sinne Gibbons $(1999,2000)$ entwickeln und gesellschaftlich robustes Wissen erzeugen.

\section{Transparenz von Beratung und Entscheidungsfindungsprozessen}

Ein weiterer relevanter Aspekt ist die Transparenz von Beratungs- und Entscheidungsfindungsprozessen sowie der Zugang zu Dokumenten und Sitzungen. Diese Faktoren sind wesentlich für die Schaffung von gesellschaftlichem Vertrauen in die Arbeit der Beratungsgremien. Die (mangelnde) Transparenz und Aspekte der Prozessoffenheit der in diesem Beitrag behandelten Gremien werden von Seiten der Anti-Atomkraft-Bewegung stark kritisiert. Um diese Kritik im Kontext zu erfassen, bietet es sich an, einen Vergleich anzustellen, inwiefern die (kritische) Öffentlichkeit in die Arbeit dieser Kommissionen eingebunden wurde. Die Treffen und Anhörungen der Enquete-Kommission etwa waren nicht-öffentlich. Der Gesamtbericht der Enquete-Kommission mit Details zu den Empfehlungen wurde der Öffentlichkeit im Jahre 1980 vom Deutschen Bundestag zugänglich gemacht. Auch die Sitzungen des AkEnd waren nicht-öffentlich und die Anhörung verschiedener Interessenvertreter*innen wurde in separaten Sitzungen durchgeführt. Für einige spezifische Themen wurden allerdings öffentliche Workshops veranstaltet und nach Angaben im Abschlussbericht wurde das Gespräch mit verschiedenen Stakeholdergruppen gesucht (AkEnd 2002: 9f.). Die nicht-öffentlichen Sitzungen der ESK dagegen stellen einen noch geschlosseneren Prozess dar. Für die interessierte Öffentlichkeit wird kaum ersichtlich, wie die Auswahl an Themen und Aufgaben bestimmt wird, was vor dem Hintergrund des Einflusses der ESK auf die Arbeit der EndKo kritisch eingeschätzt wird. 
Die Beispiele der EndKo und des NBG stellen eine deutlich positive Entwicklung in Sachen Transparenz und Zugänglichkeit von Informationen dar. Die Sitzungen der EndKo waren größtenteils öffentlich zugänglich. Dennoch ist Verfügbarkeit nicht gleichzusetzen mit Zugänglichkeit und Transparenz: Auch wenn Materialien auf der Internetseite veröffentlicht wurden, war für interessierte Kreise die Nachvollziehbarkeit der Arbeit der Kommission deutlich erschwert (vgl. endlagerdialog.de 2016, 2015e). Beispielhaft kann genannt werden, dass »keinerlei Aufarbeitung der Kommissionsdiskussionen in verständlicher und wissenschaftsgeprägter Form« vorgenommen wurde (ebd. 2015a), die meist sehr spät veröffentlichten Audiodateien nicht mit einem Inhaltsverzeichnis versehen wurden (ebd. 2015b), keine Informationen ausgegeben wurde, warum und in welcher Form Gutachten beauftragt wurden (ebd. 2015c) sowie keine Beratungsunterlagen veröffentlicht oder vollständigen Tischvorlagen für Teilnehmer*innen der Öffentlichkeit bei Sitzungen ausgeteilt wurden (ebd. 2015d, 2015e). Die Sitzungen wurden zunächst nicht transkribiert oder protokolliert, allerdings sorgten Vertreter*innen der Zivilgesellschaft für die Anfertigung und schnellstmögliche Veröffentlichung von Wortprotokollen der ersten Sitzungen (ebd. 2014). Es ist positiv zu werten, dass eine online Übertragung aller Sitzungen in Form eines Livestreams für die interessierte Öffentlichkeit ermöglicht wurde.

Auch die regelmäßigen Sitzungen des NBGs sind prinzipiell offen. Allerdings sind Teilnehmende aus der interessierten Öffentlichkeit bei diesen Sitzungen eher Beobachter*innen und die Mitglieder behalten sich auch das Recht vor, die Öffentlichkeit von bestimmten Sitzungsthemen auszuschließen, etwa wenn es um Personalien geht. Die Sitzungen werden zwar nicht online übertragen, aber protokolliert und die Protokolle zeitnah auf der Internetseite des NBGs veröffentlicht.

\section{Einflussnahme auf Entscheidungen und Policy}

Bei der Analyse der AkEnd-Empfehlungen können wir feststellen, dass diese einen beträchtlichen Einfluss auf Entscheidungsfindungsprozesse und die Policy-Ergebnisse der letzten Jahre zum Thema Entsorgung hochradioaktiver Abfälle gehabt haben. Die Arbeit und Ergebnisse der EndKo wurden in hohem Maße durch die Empfehlungen des AkEnd geprägt. So finden sich bisweilen sogar ganze Passagen, die die EndKo im Wortlaut vom AkEnd übernommen hat. Die Anwendung der AkEnd-Empfehlungen innerhalb der EndKo kann sicher zum Teil mit den zwischen ihnen existierenden personellen Überschneidungen, aber auch den gesetzlichen Vorgaben der EndKo erklärt werden.

Es ist schwer nachzuvollziehen, wie genau die ESK politische Entscheidungen im Bereich Endlagerung beeinflusst hat, aber es ist anzunehmen, dass ihre Bedeutung erheblich war bzw. ist. Jenseits der Tatsache, dass der ehemalige Vorsitzende der ESK (bis Ende 2019) ebenfalls Mitglied im AkEnd und der EndKo war, lässt die kontinuierliche Beratungstätigkeit der ESK für das Bundesumweltministerium vermuten, dass dieses Beratungsgremium große Beachtung in den Diskursen um verschiedene Fragen des Managements und der Endlagerung hochradioaktiver Abfällen fand. 
Das NBG hat, trotz seiner wichtigen Rolle im Bereich Monitoring und Einbindung der Öffentlichkeit, wenig Einfluss auf den Entscheidungsfindungsprozess: Gemäß $\$ 8$ StandAG kann das NBG die »zuständigen Institutionen jederzeit befragen und Stellungnahmen abgeben«. Im weiteren Verfahren kann das NBG dem Bundestag gegenüber Empfehlungen aussprechen, die zwar zur Kenntnis genommen werden aber nicht bindend berücksichtigt werden müssen (StandAG 2017). Auch wenn es zur kritischen Begleitung des Endlagersuchprozesses berechtigt ist, stellen seine Empfehlungen lediglich eine öffentliche Meinung dar, die in der Vergangenheit nur teilweise bei der Entscheidungsfindung berücksichtigt wurde. Dies wurde besonders deutlich bei der relativ geringen Einbeziehung der Vorschläge zur Novellierung des StandAGs aus der Bürger*innen-Anhörung im Februar 2017 durch den Bundestag. Das NBG hatte in diesem Zusammenhang Empfehlungen für die Novellierung zusammen mit interessierten Bürger*innen entwickelt. Von diesen gemeinsam erarbeiteten NBG-Empfehlungen wurden 2017 schlussendlich nur wenige marginal in das Gesetz aufgenommen (NBG Report 2018).

\section{Distanz und Unabhängigkeit zwischen Beratenden und zu Beratenen}

Hinsichtlich des Prinzips der Distanz zwischen bzw. Unabhängigkeit von Beratenden und Beratenen bedarf es einer tiefergehenden Analyse in zukünftigen Untersuchungen. Erste Anhaltspunkte liefert jedoch die zivilgesellschaftliche Kritik, besonders der Anti-Atombewegung, an der Mitgliederauswahl für die EndKo. So hätten manche Mitglieder mehrere Rollen inne. Die Anti-Atom-Organisation .ausgestrahlt argumentiert zum Beispiel, dass Vertreter*innen der Atomindustrie versuchten, ihre Interessen im EndKo-Abschlussbericht einzubringen. Die Organisation kritisierte auch die Verbindungen und die verschiedenen Rollen und Interessen von bestimmten Expert*innen. So war einer der zwei Gewerkschaftsvertreter*innen seit 2005 auch Mitglied im Aufsichtsrat des Energieversorgers E.ON AG/E.ON SE sowie seit 2010 stellvertretender Vorsitzender des Aufsichtsrats der E.ON AG/E.ON SE, obwohl bereits ein Vertreter dieses Energieunternehmens in der EndKo vertreten war. Bei einer solchen Doppelfunktion von Mitgliedern kann nicht ausgeschlossen werden, dass bestimmte Interessen Dritter vertreten werden, so dass die Unabhängigkeit entsprechender Akteure in Zweifel gerät.

Im Fall des NBGs fordert das StandAG die Unabhängigkeit jedes Mitglieds. So dürfe bspw. kein Mitglied ein ökonomisches Interesse in Bezug auf die Standortauswahl oder die Entsorgung hochradioaktiver Abfälle haben und dürfe »weder einer gesetzgebenden Körperschaft des Bundes oder eines Landes noch der Bundes- oder einer Landesregierung angehören« (StandAG 2017, \$8 (3)).

Daneben lässt sich im Vergleich der Mitglieder aller hier betrachteten Gremien feststellen, dass es zu wiederkehrenden Expert*innen kommt, die bspw. sowohl im AkEnd, als auch der EndKo und ESK vertreten waren und in einigen Fällen auch verschiedene Rollen einnahmen. Auch mit Blick auf die neu gegründeten Institutionen BGE und BASE zeigen sich Überschneidungen zu den Kommissionen. So war eine der Vorsitzenden der EndKo anschließend Vorsitzende der Geschäftsführung der Bundesgesellschaft für Endlagerung (BGE). Ebenso ist ein Mitglied der ESK Teil der Geschäftsführung der BGE 
geworden, woraufhin diese Person von ihrer Mitgliedschaft in der ESK zurückgetreten ist.

Wenn die Funktionen bzw. Mitgliedschaften sich zwischen diversen Beratungsgremien oder zwischen einem Beratungsgremium und einer Betreibergesellschaft oder einer Bundesbehörde überschneiden, so wirft dies Fragen nach der Distanz bzw. Unabhängigkeit der Berater*innen auf. Dies führt oftmals zu öffentlicher Kritik und somit einer verminderten politischen Robustheit in der Entscheidungsfindung.

In der Abb. 3 werden unsere Ergebnisse zusammengeführt und eine vergleichende Gegenüberstellung der untersuchten Gremien hinsichtlich der »Prinzipien guter Beratung«

Abbildung 3: Bewertung ausgewählter Beratungsgremien im Endlagerungskontext unter der Berücksichtigung der Prinzipien guter wissenschaftlicher Politikberatung vorgenommen.

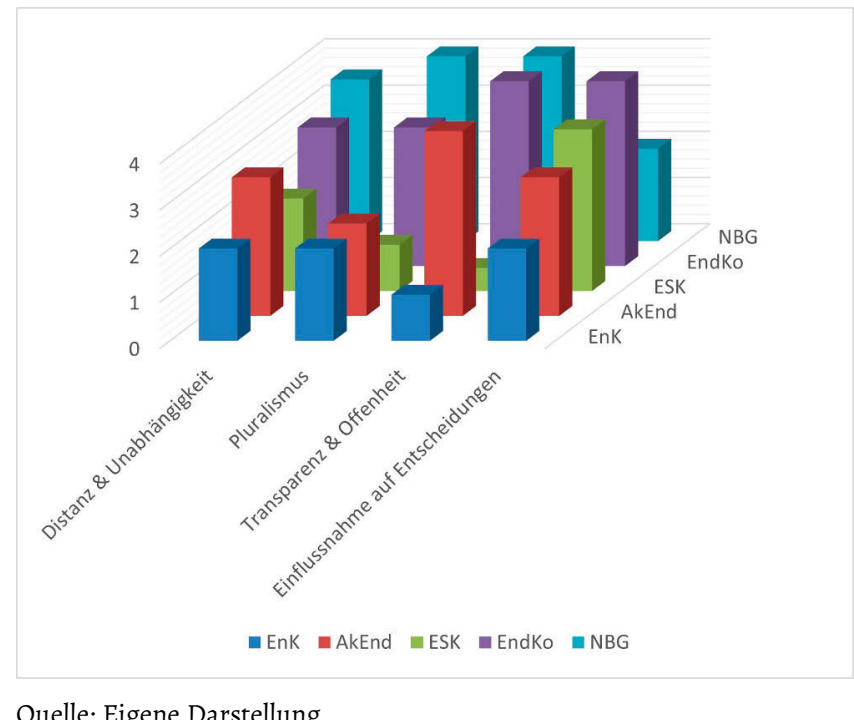

Quelle: Eigene Darstellung

\section{Fazit}

Unsere Analyse verdeutlicht eine langsame, aber stetige Veränderung in der Zusammensetzung und in den Schwerpunktsetzungen der Beratungsgremien zur Thematik der Endlagerung hochradioaktiver Abfälle über den Zeitraum von 1979 bis zur Gegenwart. Dieser Wandel spiegelt sich vor allem in der Zusammensetzung des neuesten Gremiums, dem NBG, sowie der stetig wachsenden Relevanz des Aspekts einer aktiven und befähigenden Öffentlichkeitsbeteiligung im Zusammenhang mit dem Standortauswahlverfahren in den Empfehlungen der Enquete-Kommission, des AkEnd, der EndKo und des NBGs. 
Im Vergleich zur Enquete-Kommission, der ESK und dem AkEnd, verdeutlicht die Mitgliederstruktur der EndKo wie auch die des NBGs die zunehmende politische Relevanz gesellschaftlicher Belange in den vergangenen zwei Dekaden. In diesem Zusammenhang hat sich die Struktur der Gremien verändert, um den Erwartungen der Gesellschaft besser gerecht zu werden. Ferner lässt sich eine Verbesserung im Geschlechterverhältnis beobachten sowie im Verhältnis zwischen wissenschaftlichen und nichtwissenschaftlichen Beratungsmitgliedern. Waren die AkEnd-Mitglieder allesamt allein aufgrund ihrer naturwissenschaftlichen Expertise berufen worden, zeigt sich in der Zusammensetzung der EndKo bereits eine deutliche Diversität in den Disziplinen und dem Pluralismus an Stakeholdergruppen. Durch die verstärkte Offenheit hinsichtlich Pluralismus und Vielfalt der Wissensformen wird auch ein zunehmend robusteres epistemisches Wissen erzeugt, mit Blick auf Entscheidungen, in denen per se Unsicherheiten vorhanden sind und Wissen umkämpft ist (vgl. auch den Beitrag von Chaudry und Seidl sowie den Beitrag von Themann in diesem Sammelband).

Das NBG stellt hierbei eine aussichtsvollere Annäherung an das dar, was Gibbons (1999) socially robust knowledge nannte. Dabei entsteht das Paradoxon, dass das Gremium, das am stärksten nicht-wissenschaftliche Expertise in den Prozess einzubringen versucht, den geringsten Einfluss auf Entscheidungsprozesse hat. Hingegen scheinen alte Empfehlungen, die auf weniger pluralen und disziplinär wenig diversen Gremien beruhen, einen wesentlich höheren Einfluss auf den derzeitigen Prozess und seine Ziele zu haben als neue Gremien, wie der nachhaltige Einfluss des AkEnd zeigt.

Doch das wicked problem der Endlagerung, das uns mit unknown unknowns konfrontiert (vgl. Themann und Brunnengräber 2019) und entsprechenden wissenschaftlichen Unsicherheiten und Risiken, weist auf die ethische Dimension der Entscheidungen in diesem Zusammenhang hin. Ein rein naturwissenschaftlicher und technischer Zugang zur Entscheidungsfindung im Zusammenhang mit Hochrisikotechnologien sind nicht mehr angemessen. Beck (1995) hat mit seinen Theorien zur Risikogesellschaft bzw. reflexiven Modernisierung also bereits auf die zunehmend relevante Rolle von Laien bzw. Individuen hingewiesen, die sich angesichts von Hochrisikotechnologien und Unsicherheiten und der damit verbundenen ethischen Fragen Wissen und Expertise aneignen (müssen). Den gesamtgesellschaftlichen Dialog und den demokratischen Streit (Römmele 2019) gilt es neu zu kultivieren. Die noch sehr verhaltene Reaktion auf die Impulse durch das NBG verdeutlichen, dass unsere derzeitigen Entscheidungsfindungsprozesse noch nicht gänzlich bereit für Strukturen und Institutionen sind, in denen Wissenschaft und Gesellschaft eine neue Form des Diskurses praktizieren und sich darauf vorbereiten, gegenseitig voneinander zu lernen.

\section{Literatur}

AkEnd (2002): Auswahlverfahren für Endlagerstandorte. Empfehlungen des AkEnd Arbeitskreis Auswahlverfahren Endlagerstandorte. https://www.bundestag.de/en dlager-archiv/blob/2819 06/c1fb3860506631de51b9fif689b7664c/kmat_01_akend-data.pdf, zuletzt geprüft am 03.08.2020. 
atommüllreport (2020): Nationales Begleitgremium, https://www.atommuellreport.d e/themen/endlagerung/einzelansicht/nationales-begleitgremium.html, zuletzt geprüft am 03.08.2020.

atommüllreport (2016): Enquete-Kommission Zukünftige Kernenergiepolitik. https:// www.atommuellreport.de/themen/kommissionen/einzelansicht/enquete-kommis sion-zukuenftige-kernenergiepolitik.html, zuletzt geprüft am 03.08.2020.

.ausgestrahlt (2014): Quasi unter Ausschluss der Öffentlichkeit: Die vierte Sitzung der Atommüll-Kommission. https:/www.ausgestrahlt.de/blog/2014/10/05/quasi-unter -ausschluss-der-offent lichkeit-die-vier/, zuletzt geprüft am 03.08.2020.

Beck, Ulrich (1995): Judo-Politik. Über die Entstehung supranationaler Öffentlichkeiten und die Chancen der Subpolitik, über Greenpeace als Agentur des inszenierten Konflikts und die neue Wichtigkeit politischer Symbole anhand der Affäre »Brent Spar«. In: die tageszeitung vom 1./2.07.1995, 13/14.

Becker, Torben (2017): Neues Standortauswahlgesetz schwächelt. https://www.bund.n et/themen/aktuelles/detail-aktuelles/news/neues-standortauswahlgesetz-schwaec helt/, zuletzt geprüft am 03.08.2020.

Bergmans, Anne; Sundqvist, Göran; Kos, Drago; Simmons, Peter (2015): The participatory turn in radioactive waste management: Deliberation and the social-technical divide. Journal of Risk Research 18/3: 347-363.

Boswell, Christina (2012): How Information Scarcity Influences the Policy Agenda: Evidence from U.K. Governance (25:3), 367-389.

Cozzens, Susan E.; Woodhouse, Edward J. (1995): Science, Government, and the Politics of Knowledge. In: Jasanoff, Sheila; Markle, Gerald E.; Peterson, James C. and Pinch, Trevor (Eds.) (1995). Handbook of Science and Technology Studies, Thousand Oaks: Sage Publications, 532-553.

Deutscher Bundestag (2019a): Kommissionen. Online: https://www.bundestag.de/serv ice/glossar\#url=L3NlcnZpY2UvZ2xvc3Nhci9nbG9zc2FyLosva29tbWlzc2lvbmVuLzI ONTQ4MA==\&mod=mod445382, zuletzt geprüft am 03.08.2020.

Deutscher Bundestag (2019b): Enquete-Kommission. https://www.bundestag.de/servi ce/glossar\#url=L3NlcnZpY2UvZ2xvc3Nhci9nbG9zc2FyLoUvZW5xdWVoZS80NDQ $3 \mathrm{MzQ}=\& \bmod =\bmod 445382$, zuletzt geprüft am 03.08.2020.

Deutscher Bundestag (2017): Geschäftsordnung des Deutschen Bundestages. https:// www. bundestag.de/parlament/aufgaben/rechtsgrundlagen/go_btg, zuletzt geprüft am 03.08.2020.

Deutscher Bundestag (1980): Bericht der Enquete Kommission »Zukünftige Kernenergiepolitik«. http://dip21.bundestag.de/dip21/btd/08/043/0804341.pdf, zuletzt geprüft am 03.08.2020.

Di Nucci, Maria Rosaria; Pearce, David A. (1989): Technology vs. Science: The Cognitive Fallacy. In: Synthese, 81(3), 405-419.

Douglas, Heather E. (2009): Science, policy, and the value-free ideal. Pittsburgh University Press.

EndKo (2016): Abschlussbericht. Verantwortung für die Zukunft. Ein faires und transparentes Verfahren für die Auswahl eines nationalen Endlagerstandortes. https://www.bundestag.de/endlager-archiv/blob/434430/bb37b21b 8e1e7e049ace5db6b2f949b2/drs_268-data.pdf, zuletzt geprüft am 03.08.2020. 
endlagerdialog.de (2014): .ausgestrahlt macht die Arbeit der Endlagerkommission transparenter. https://endlagerdialog.de/2014/08/ausgestrahlt-macht-arbeit-endla gerkommission-transparenter/

endlagerdialog.de (2015a): Endlagerkommission: Wie kann die Arbeit doch noch zu einem Erfolg führen? https://endlagerdialog.de/2015/12/endlagerkommission-wie-ar beit-noch-erfolg-fuehren/\#more-7349

endlagerdialog.de (2015b): Sitzung AG 1: Vom Dunkel einer Ablehnung zum Lichtschimmer am Horizont. https://endlagerdialog.de/2015/11/sitzung-ag-1-vom-dunkel-able hnung-lichtschimmer-horizont/

endlagerdialog.de (2015c): $¿$ »Wir wollen Beteiligen lernen!« ? https://endlagerdialog.de /2016/01/wir-wollen-beteiligen-lernen/\#more-7457

endlagerdialog.de (2015d): Endlagerkommission: Wenig Interesse am Leitbild. https:// endlagerdialog.de/2015/09/endlagerkommission-wenig-interesse-leitbild/\#more-7 216

endlagerdialog.de (2015e): Vom endgültigen Scheitern der Transparenz. https://endlag erdialog.de/2015/03/vom-endgueltigen-scheitern-transparenz/

endlagerdialog.de (2016): AG 3: Langsam, langsam wächst die Transparenz und die Länderkompetenz wird einbezogen. https://endlagerdialog.de/2016/02/ag-3-langsamlangsam-waechst-transparenz-laenderkompetenz-einbezogen/\#more-7470

ESK (2015): Entsorgungskommission (ESK). www.entsorgungskommission.de/de/hom e, zuletzt geprüft am 03.08.2020

Gibbons, Michael (1999): Science's new social contract with society. In: Nature 402, 8184 .

Gibbons, Michael (2000): Mode 2 society and the emergence of context-sensitive science, in: Science and Public Policy, 27(39), 159-163.

Guston, David H. (2000): Between politics and science: Assuring the integrity and productivity of research. New York: Cambridge University Press.

Jasanoff, Sheila (1990): The fifth branch: science advisers as policymakers. Cambridge, MA: Harvard University Press.

Jasanoff, Sheila (2004): States of Knowledge: The Co-Production of Science and the Social Order. London: Routledge.

Jasanoff, Sheila (2011): Quality control and peer review in advisory science. In: Lentsch, J. and Weingart, P. (Eds.) (2011). The Politics of Scientific Advice: Institutional Design for Quality Assurance. Cambridge: Cambridge University Press, 19-35.

Kruglanski, Arie W. (1980): Lay epistemo-logic-Process and contents: Another look at attribution theory. Psychological review, 87, 70-87.

Lentsch, Justus; Weingart, Peter (2011): Introduction: the quest for quality as a challenge to scientific policy advice: an overdue debate? In: Lentsch, Justus; Weingart, Peter (Eds.) (2011). The Politics of Scientific Advice: Institutional Design for Quality Assurance. Cambridge: Cambridge University Press, 3-18.

Maasen, Sabine; Weingart, Peter (2005): What's new in scientific advice to politics? In: Maasen, S. and Weingart, Peter (Eds.) (2005): Democratization of Expertise? Exploring Novel Forms of Scientific Advice in Political Decision-Making, Dodrecht: Springer, 1-20. 
NBG (2016): Die Aufgaben. Online: www.nationales-begleitgremium.de/DE/Gremium/WasWirMachen/WasWirMachen_node.html, zuletzt geprüft am 03.08.2020.

NBG Report (2018): Erster Bericht zum Auswahlverfahren für einen Endlagerstandort. www.nationales-begleitgremium.de/SharedDocs/Downloads/DE/Downloads_17.Sitzung _15.05.2018/1.T\%C3\%A4tigkeitsbericht_NBG.pdf?_blob=publicationFile\&v=14, zuletzt geprüft am 03.08.2020.

NBG (2020): Das Gremium. https://www.nationales-begleitgremium.de/DE/WerWirS ind/Das_Gremium/Das_Gremium_node.html, zuletzt geprüft am 03.08.2020.

Pedersen, David B. (2014): The Political Epistemology of Science-Based Policy-Making. In: Social Science and Public Policy, 51, 547-551.

Popper, Karl (1963): Conjectures and Refutations: The Growth of Scientific Knowledge, 5th edition, London: Routledge.

Römmele, Andrea (2019): Zur Sache! Für eine neue Streitkultur in Politik und Gesellschaft. Aufbau Verlag, Berlin.

Siefken, Sven T. (2007): Expertenkommissionen im politischen Prozess. Eine Bilanz zur rot-grünen Bundesregierung 1998 - 2005. Wiesbaden: VS Verlag.

StandAG - Standortauswahlgesetz(2017): Novellierung-Gesetz zur Suche und Auswahl eines Standortes für ein Endlager für hochradioaktive Abfälle. https://ww w.gesetze-im-internet.de/standag_2017/BJNR107410017.html, zuletzt geprüft am 03.08.2020.

StandAG (2013): Gesetz zur Suche und Auswahl eines Standortes für ein Endlager für hochradioaktive Abfälle. www.bgbl.de/xaver/bgbl/start.xav?startbk=Bundesanzeiger_BGBI\&jumpTo=bgbli13s2553.pdf, zuletzt geprüft am 03.08.2020.

Stay, Jochen (2019): Das Verfahren vom Kopf auf die Füße stellen Oder: Was brauchen die Betroffenen, um zustimmen zu können? Fachgespräch der Bundestagsfraktion Die Linke 22. März 2019 Berlin, https://www.hubertus-zdebel.de/wp-conte nt/uploads/2019/03/Vortrag-Stay-Standortsuche-22.3.2019.pdf, zuletzt geprüft am 03.08.2020.

Themann, Dörte; Brunnengräber, Achim (2019): The nuclear legacy in the Anthropocene: interrelation between nature, technology and society, in: Hickmann, Thomas; Partzsch, Lena; Pattberg, Philipp; Weiland, Sabine (Hg.) (2019): The Anthropocene Debate and Political Science, London and New York: Routledge, Research in Global Environmental Governance, 182-199.

Van den Hove, Sybille (2007): A rationale for science-policy interfaces. In: Futures, 39(7), S. 807-826.

Volkmann, Ute (2007): Das schwierige Leben in der »Zweiten Moderne« — Ulrich Becks »Risikogesellschaft«. In: Schimank, Uwe; Volkmann, Ute (Hg.): Soziologische Gegenwartsdiagnosen I. VS Verlag für Sozialwissenschaften. 23-40.

Zürn, Michael (2012): Autorität und Legitimität in der postnationalen Konstellation, in: Geis, Anna; Nullmeier, Frank; Daase, Christopher (Hg.): Der Aufstieg der Legitimitäts-politik. Rechtfertigung und Kritik politisch-ökonomischer Ordnungen (Leviathan-Sonderband 27/2012), Baden- Baden: Nomos, 41-62. 